\title{
Anemia durante el tercer trimestre del embarazo, Cuenca - Ecuador
}

\section{Anemia during the third quarter of pregnancy, Cuenca - Ecuador}

\author{
María Cecilia Ochoa Hurtado ${ }^{1,2}$, Julio César Cárdenas Mateus ${ }^{1,2}$, Jeanneth Patricia Tapia Cárdenas ${ }^{* 3}$, Zoila \\ Katherine Salazar Torres ${ }^{3}$, Edison Gustavo Moyano Brito ${ }^{3}$ \\ ${ }^{1}$ Ministerio de Salud Pública, Ecuador \\ ${ }^{2}$ Hospital del Instituto Ecuatoriano de Seguridad Social \\ ${ }^{3}$ Universidad Católica de Cuenca, Ecuador \\ *jptapiac@ucacue.edu.ec
}

DOI: https://doi.org/10.26871/killkanasalud.v4i4.755

\begin{abstract}
Resumen
Antecedentes: La anemia en la actualidad es considerada como un problema de salud a nivel mundial, con mayor frecuencia en países en vías de desarrollo, con un incremento en la morbi-mortalidad materno fetal. Objetivo: Determinar la prevalencia de la anemia y factores asociados durante el tercer trimestre del embarazo, en el Departamento de Ginecología-Obstetricia del Hospital Vicente Corral Moscoso, 2018. Materiales y métodos: Se realizó un estudio observacional, analítico, transversal, con la participación de 354 embarazadas que cumplieron los criterios de inclusión. La información se recolectó de los expedientes clínicos mediante un formulario. Para el análisis estadístico se empleó el programa SPSS versión 15, estadística descriptiva, y para la asociación de factores de riesgo se aplicó la prueba chi cuadrado, reportándose una razón de prevalencia con nivel de confianza del $95 \%$ y el valor de p $(<0.05)$. Resultados: La prevalencia de anemia fue del 25,7\% (IC 95\%: 21.01\%-30.4\%) siendo más elevada en adultas jóvenes con el 40,7\%, residentes en áreas rurales con $51,6 \%$, instrucción secundaria $62,6 \%$, ocupación ama de casa con $46,2 \%$, estado civil soltera y casada en un $40 \%$; además fue más común en gestantes con $>5$ controles prenatales con el 74,7\%, multigestas en un $62,6 \%$, embarazos a término con el $86,8 \%$. Además, se encontró relación estadísticamente significativa $(\mathrm{p}<0,05)$ con: adultas jóvenes y primigestas; el sobrepeso u obesidad fueron un factor protector. Conclusiones: La prevalencia de anemia fue elevada y se asoció con factores no modificables como: edad y ser primigesta.
\end{abstract}

Palabras clave: anemia, epidemiología, diagnóstico, embarazo .

\begin{abstract}
Background information: At present, anemia is considered a health problem worldwide, predominating at its most part in developing countries, and causing an increase in maternal-fetal morbimortality. Objective: To determine the prevalence of the anemia and associated factors during the third trimester of pregnancy in the Gineco-Obstetrics Department, in Vicente Corral Moscoso Hospital in 2018. Materials and methods: Observational, analytical, and transversal studies were conducted in this research, where 354 patients contributed and complied with the criteria for their inclusion in this study. The information was extracted from clinical files by means of a form. For the statistical analysis, version 15 of the program SPSS was utilized, making use of descriptive statistics; for the association of risk factors, the Chi-square test was applied, reporting predominance with a level of reliability of $95 \%$, and the value of $(<0.05)$. Results: The prevalence of anemia was 25,7\% (IC 95\%: 21.01\%-30.4\%) being predominantly higher in female young adults with 40,7\%; rural areas inhabitants with $51,6 \%$, secondary education with $62,6 \%$, those with an occupation of housewife with a 46,2\%, and civil status single and married respectively, with a $40 \%$. In addition, it was common in pregnant women with $>5$ prenatal control with 74,7\%; multiple gestations in a 62,6\%, and pregnancy end periods with 86,8\%. It was found a statistically significant relation $(p<0,05)$ between young adults and newly pregnants; overweighting and obesity were protective factors. Conclusions: The prevalence of anemia was high, and it was linked to non-alterable factors such as age, and the fact of being newly pregnant.
\end{abstract}

Keywords: anemia, epidemiology, diagnosis, pregnancy .

\section{Introducción}

Se considera anemia a la disminución del nivel de hemoglobina ocasionada por el déficit de glóbulos rojos destinados al transporte de oxígeno hacia las células. ${ }^{1}$ Fisiológicamente durante la gestación existe un incremento en el plasma sanguíneo además de los hematíes, que ayudan 
a compensar los diversos cambios funcionales del embarazo. $^{2}$

La anemia gestacional puede causar complicaciones, que, si no son detectadas durante el periodo prenatal y tratadas de manera adecuada conllevan un riesgo para desarrollar hemorragia postparto, amenaza de parto pretérmino además de consecuencias no favorables para el binomio madre e hijo?,?

La Organización Mundial de la Salud (OMS) ${ }^{5}$ y el Colegio Estadounidense de Obstetras y Ginecólogos (ACOG), clasifican a la anemia según los niveles de hemoglobina y de acuerdo con con el trimestre del embarazo en los siguientes rangos: nivel inferior a $11 \mathrm{~g} / \mathrm{dl}$ en el primer y tercer trimestre y $10.5 \mathrm{~g} / \mathrm{dl}$ en el segundo. ${ }^{6}$

En el Ecuador según las normas del Ministerio de Salud Pública (MSP), se clasifica la anemia de acuerdo al nivel de gravedad en: leve, moderada y severa en dependencia de la concentración de hemoglobina con rangos que oscilan entre $10.1-10.9 \mathrm{~g} / \mathrm{dl} ; 7-10 \mathrm{~g} / \mathrm{dl}$ e inferior a $7 \mathrm{~g} / \mathrm{dl}^{5}$ respectivamente. ${ }^{7}$

La génesis de la anemia durante la gestación es multifactorial y está relacionada con la edad, ocupación, paridad, nivel de instrucción, residencia, antecedentes obstétricos, número de controles prenatales y el seguimiento de la gestación durante cada trimestre. ${ }^{8,9}$

La Organización Mundial de la Salud indica una prevalencia del $41,8 \%$ para anemia en la gestación siendo la causa principal la deficiencia de hierro, ${ }^{5}$ ésta realidad tiene mayor contraste en grupos de madres con menor nivel de instrucción, bajo estatus socioeconómico, cursar su tercer trimestre de la gestación y un mal manejo preconcepcional $(10,11)$.

En la literatura biomédica se puede evidenciar que las cifras de anemia varían en dependencia de las áreas geográficas, y el tamaño muestral de los diferentes estudios consultados, es así que en Europa se muestran frecuencias de alrededor del 27,5\%, Asia y otras Islas del Pacífico un $29 \%$, en afroamericanas el $48,5 \%$, en americanas un $33,9 \%$, en hispanas y latinas en un $30,1 \% .^{12}$

Un metaanálisis realizado en China con 5.8752 embarazadas determinó que la prevalencia de anemia durante la gestación fue $19,9 \%$ y en el tercer trimestre de $28,1 \%{ }^{13}$ En Etiopía en el año 2017, se determinó la prevalencia de anemia en un $23 \%$ y conforme se analizó los factores que se relacionaron con el nivel socio económico, el cursar el tercer trimestre del embarazo, multiparidad, bajo acceso a cuidados prenatales y malnutrición. ${ }^{14}$

En Colombia en el 2010, en un estudio donde participaron 1.385 gestantes entre 13 y 49 años, la prevalencia anemia fue del $11 \%$; los factores asociados fueron: nivel de instrucción en $19.4 \%$, bajo nivel socioeconómico en $12,7 \%$, residencia región atlántica o pacífica $16 \%$ y $12.7 \%$ respectivamente y mujeres afro-colombianas en $18,6 \%{ }^{15}$

En Perú en un estudio se incluyó 28.7691 registros, obteniéndose una prevalencia del $28 \%$, siendo la anemia leve la más frecuente con el 25,1\%. ${ }^{16}$ En México se evidenció una prevalencia de anemia del $20,6 \% .^{17}$

En Pereira, en un estudio realizado a 1.493 mujeres se demostró una prevalencia de anemia del 32,01\%. ${ }^{18}$ En Bolivia, en una investigación se revisaron 98 historias clínicas, la prevalencia de anemia en la gestación fue de $40.8 \%$ y según la gravedad leve, para moderada y grave fue de $65,3 \%$, $28,7 \% 5.9 \% .^{19}$ En Venezuela en 520 gestantes se presentó una prevalencia de anemia ferropénica de $51,3 \% .^{20}$

En Ecuador, existen diferentes reportes de la incidencia de anemia en gestantes; es así que en el 2012 en el Hospital Isidro Ayora en embarazadas que cursaban el tercer trimestre de gestación la prevalencia fue del $12 \%,{ }^{21}$ en un estudio descriptivo en la ciudad de Cuenca en el mismo año se evidenció una frecuencia del $12 \% .^{22}$ La Guía de Práctica Clínica del MSP, indicó un ascenso en la frecuencia de anemia a 46,9\%; en esta línea, una investigación realizada en Quito encontró una prevalencia de anemia del $46,5 \%$. $^{23}$

Otros estudios identificaron como factores de riesgo el nivel socioeconómico, ser madres adolescentes, sin pareja, bajo estado nutricional y etnia, no obstante, esta patología es evidentemente prevenible y manejable $(24,25)$.

Existen múltiples causas que pueden llevar al desarrollo de anemia, por lo que se debe realizar un control adecuado para evitar complicaciones y de la misma manera iniciar un tratamiento precoz; ${ }^{26}$ sin embargo, durante el embarazo se describen con mayor frecuencia la anemia asociada a la deficiencia de hierro y la fisiológica o dilucional; las otras causas deberán estudiarse en pacientes que tengan embarazos de alto riesgo. ${ }^{27}$

De acuerdo a lo descrito, al iniciar la gestación ocurrirán cambios fisiológicos de adaptación del organismo que de una $\mathrm{u}$ otra forma pueden contribuir a que se presenten ciertas complicaciones, por lo que el objetivo de la presente investigación es determinar la prevalencia de la anemia y factores asociados durante el tercer trimestre del embarazo, en el Departamento de Ginecología-Obstetricia del Hospital Vicente Corral Moscoso, 2018.

\section{Materiales y metodos}

Se realizó un estudio observacional, analítico, transversal, el universo del estudio lo conformaron 4776 historiales clínicos de las pacientes que cursaron el tercer trimestre del embarazo que ingresaron al Área de Ginecología y Obstetricia del Hospital Vicente Corral Moscoso durante el año 2018. Para el cálculo el tamaño de la muestra se consideró sobre la base del $95 \%$ del nivel de confianza, $5 \%$ de error de inferencia y una prevalencia de anemia del $46.9 \%$ según la Guía de Práctica Clínica del 2014, ${ }^{28}$ obteniendo una muestra de 354 pacientes. Para dar representatividad a la muestra, se realizó un muestreo probabilístico aleatorio simple de las historias clínicas que cumplieron los criterios de inclusión. 
Para el análisis de los datos se procedió conforme al tipo de variable, para las variables dico o politómicas se utilizó frecuencias y porcentajes, para las cuantitativas medidas de tendencia central como la media, y de dispersión como la desviación estándar (DS). Para la determinación de la asociación entre los factores y la anemia se procedió inicialmente aplicando la prueba de Chi Cuadrado de Pearson (X2) y para cuantificar el riesgo se empleó la razón de prevalencia (RP), nivel de confianza de $95 \%$, para la determinación de la significancia estadística se usó el valor de $\mathrm{p}$ con un punto de cohorte en 0,05 (5\%).

La información recolectada se realizó a través del historial clínico que fue de manejo exclusivo para el presente estudio, con carácter de confidencialidad, por lo que la identidad de las pacientes se mantuvo en absoluta reserva por parte de la investigadora, los formularios fueron anónimos y con codificación, los datos adquiridos fueron utilizados únicamente para el desarrollo de la investigación, sin existir conflicto de interés.

\section{Resultados}

La tabla 1 muestra las características demográficas de la población, la media de la edad se ubicó en 26 años \pm (DS) 4,3 , siendo el grupo de adultas jóvenes el más prevalente con el 55,4\%, residentes en el área rural con el 52,3\%. Más de la mitad de la población se dedicaba a tareas domésticas, de instrucción secundaria en el $57,6 \%$, y en el $40,7 \%$ fueron solteras.

Tabla 1: Características sociodemográficas de 354 embarazadas del HVCM. Cuenca, 2018

\begin{tabular}{lcc}
\hline Característica & $\mathbf{n = 3 5 4}$ & $\mathbf{\% = 1 0 0}$ \\
\hline Edad * & 149 & 42,1 \\
\hline Juventud plena & 196 & 55,4 \\
Adulta joven & 9 & 2,5 \\
Adulta madura & & \\
\hline Residencia & 169 & 47,7 \\
\hline Urbana & 185 & 52,3 \\
Rural & & \\
\hline Ocupación & 58 & 16,4 \\
\hline Estudiante & 183 & 51,7 \\
QQDD & 11 & 3,1 \\
Empleada privada & 5 & 1,4 \\
Empleada publica & 97 & 27,4 \\
Otros & & \\
\hline Instrucción & 0,6 & \\
\hline Ninguna 2 & 113 & 31,9 \\
Primaria & 204 & 57,6 \\
Secundaria & 35 & 9,9 \\
Superior & & \\
\hline Estado civil & 144 & 40,7 \\
\hline Soltera & 136 & 38,4 \\
Casada & 12 & 3,4 \\
Divorciada & 59 & \\
Unión de hecho & & \\
Viuda & & \\
\hline Edad: media 26 años desviación estándar & 4,3 \\
Juventud plena (20-24 años) & & \\
Adulta joven (25-34 años) & & \\
Adulta madura (35-65años) & & \\
& & \\
\hline
\end{tabular}

En la tabla 2, el 73,2\% de la población se realizaron $>5$ controles prenatales, la edad gestacional el $87,6 \%$ fueron a término con una media de 38,5 \pm (DS) 1,9 y el $72,3 \%$ multigestas. En cuanto al estado nutricional, la media del IMC fue de $29 \pm$ (DS) 4,2 y el 44,9\% de pacientes tuvieron sobrepeso.

Tabla 2: Características obstétricas y nutricionales de 354 embarazadas del HVCM. Cuenca, 2018

\begin{tabular}{|c|c|c|}
\hline Característica & $\mathrm{n}=354$ & $\%=100$ \\
\hline \multicolumn{3}{|c|}{ Número de controles prenatales } \\
\hline$<5$ controles & 95 & 26,8 \\
\hline$>5$ controles & 259 & 73,2 \\
\hline \multicolumn{3}{|l|}{ Edad gestacional * } \\
\hline Muy pretérmino & 4 & 1,1 \\
\hline Pretérmino moderado & 17 & 4,8 \\
\hline Pretérmino tardío & 23 & 6,5 \\
\hline A término & 310 & 87,6 \\
\hline \multicolumn{3}{|l|}{ Gestación } \\
\hline Primigesta & 98 & 27,7 \\
\hline Multigesta & 256 & 72,3 \\
\hline \multicolumn{3}{|l|}{ Estado nutricional** } \\
\hline Bajo peso & 2 & 0,6 \\
\hline Normal & 54 & 15,3 \\
\hline Sobrepeso & 159 & 44,9 \\
\hline Obesidad & 139 & 39,3 \\
\hline $\begin{array}{l}\text { *Muy pretérmino: } 28 \text { a } 31 \\
\text { *Pretérmino moderado: } 3 \\
\text { *Pretérmino tardío: } 34 \text { a } 3 \\
\text { * A término } 37 \text { a } 41.6 \text { sen }\end{array}$ & $\begin{array}{l}\text { de gesta } \\
\text { nanas de } \\
\text { as de gest } \\
\text { stación. }\end{array}$ & \\
\hline
\end{tabular}

La tabla 3 nos indica que la prevalencia de anemia en la población fue de $25,7 \%$ correspondiente a 91 pacientes, con un intervalo de confianza de $21.04 \%-30.4 \%$.

Tabla 3: Características obstétricas y nutricionales de 354 embarazadas del HVCM. Cuenca, 2018

\begin{tabular}{lccc}
\hline Anemia & n & \% & IC 95 \% \\
\hline Si & 91 & 25,7 & $21.04 \%-30.4 \%$ \\
No & 263 & 74,3 & \\
Total & 354 & 100 & \\
\hline
\end{tabular}

La tabla 4 demuestra como la prevalencia de anemia varía dependiendo de las características sociodemográficas, siendo más elevada en las mujeres adultas jóvenes con el $40,7 \%$, de procedencia rural $51,6 \%$, con instrucción secundaria 62,6\%, ocupación QQDD con el 46,2\% y de estado civil soltera y casada con el $40 \%$.

Tabla 4: Distribución de anemia según las características sociodemográficas de 91 embarazadas del HVCM, Cuenca, 2018

\begin{tabular}{lcc}
\hline & $\mathbf{n = 9 1}$ & $\mathbf{\% = 1 0 0}$ \\
\hline Edad & & \\
\hline Juventud plena & 51 & 56 \\
Adulta joven & 37 & 40,7 \\
Adulta madura & 3 & 3,3 \\
\hline
\end{tabular}


Tabla 4: Distribución de anemia según las características sociodemográficas de 91 embarazadas del HVCM, Cuenca, 2018...(continuación)

\begin{tabular}{lcc}
\hline & $\mathbf{n = 9 1}$ & $\mathbf{\% = 1 0 0}$ \\
\hline Residencia & & \\
\hline Rural & 47 & 51,6 \\
Urbana & 44 & 48,4 \\
\hline Instrucción & & \\
\hline Ninguna & 0 & 0 \\
Primaria & 26 & 28,6 \\
Secundaria & 57 & 62,6 \\
Superior & 8 & 8,8 \\
\hline Ocupación & & \\
\hline Estudiante & 17 & 18,7 \\
QQDD & 42 & 46,2 \\
Empleada privada & 3 & 3,3 \\
Empleada pública & 1 & 1,1 \\
Otros & 28 & 30,8 \\
\hline Estado civil & & \\
\hline Soltera & 40 & 44 \\
Casada & 40 & 44 \\
Divorciada & 2 & 2,2 \\
Unión de hecho & 9 & 9,9 \\
Viuda & 0 & 0 \\
\hline
\end{tabular}

En la tabla 5 se determinó mayor frecuencia de anemia en las mujeres que presentaron más de 5 controles prenatales con el $74,7 \%$, con en embarazos a término el $86,8 \%$, multigestas con el $62,6 \%$, que no presentaron abortos, cesáreas ni partos vaginales, y el $40.7 \%$ presentó sobrepeso.

Tabla 5: Distribución de anemia según los factores obstétricos y nutricionales de 91 embarazadas del HVCM. Cuenca, 2018

\begin{tabular}{lcc}
\hline & $\mathbf{n = 9 1}$ & $\mathbf{\% = 1 0 0}$ \\
\hline Controles prenatales & & \\
\hline$\leq 5$ & 23 & 25,3 \\
$>5$ & 68 & 74,7 \\
\hline Edad gestacional & & \\
\hline Muy pretérmino & 1 & 1,1 \\
Pretérmino moderado & 5 & 5,5 \\
Pretérmino tardío & 6 & 6,6 \\
A término & 79 & 86,8 \\
\hline Gestación & & \\
\hline Primigesta & 34 & 37,4 \\
Multigesta & 57 & 62,6 \\
\hline Aborto & & 12,1 \\
\hline Si & 11 & 87,6 \\
No & 80 & 24,2 \\
\hline Cesárea & & 75,8 \\
\hline Si & 22 & \\
No & 69 & 46,2 \\
\hline Parto vaginal & & 53,8 \\
\hline Si & 42 \\
No & 49 & 22 \\
\hline Estado nutricional & & \\
\hline Normal & 20 & \\
Sobrepeso & 37 & \\
Obesidad & 34 & \\
\hline
\end{tabular}

En la tabla 6 del total de la población con anemia el $76,9 \%$ presentó anemia leve y el tipo morfológico más frecuente fue la normocítica con el 90,1\%. La media de concentración de hemoglobina fue de 12,3 \pm (DS) 1,5 y de hematocrito 36,4 \pm (DS) 4,8. La media de VCM fue de $84,78 \pm($ DS) 3,44 .

Tabla 6: Clasificación de anemia según su gravedad y morfología de 91 embarazadas del HVCM. Cuenca, 2018

\begin{tabular}{lcc}
\hline Clasificación & n & \% \\
\hline Leve & 70 & 76,9 \\
Moderada & 19 & 20,9 \\
Severa & 2 & 2,2 \\
Total & 91 & 100 \\
\hline Clasificación Morfológica* & & \\
\hline Microcítica & 8 & 8,8 \\
Normocítica & 82 & 90,1 \\
Macrocítica & 1 & 1,1 \\
Total & 91 & 100 \\
\hline *Media VCM $84,78 \pm 3,44$ & &
\end{tabular}

En la tabla 7 se observan los factores de riesgo analizados en el presente estudio, donde se consideró la juventud como factor de riesgo, se encontró que este grupo de edad aumentó el riesgo de anemia en 1,7 veces (IC 95\%: 1,22,5; valor p:0,00). Por otro lado, las pacientes primigestas mostraron 1,5 veces más riesgo de anemia en comparación con las multigestas (IC 95\%: 1-2,1; valor p:0,01).

Además, la procedencia rural, nivel de instrucción primaria y ninguna, poseer menos de 5 controles, gestación a término, aborto, cesárea y parto vaginal no fueron considerados factores de riesgo estadísticamente significativos.

El sobrepeso/obesidad, resultó ser un factor protector pues solamente el $23.8 \%$ de gestantes con sobrepeso u obesidad presentaron anemia (RP: 0,6; IC 95\%: 0,4-0,9; valor p:0,04). 
Tabla 7: Factores de riesgo asociados en las 91 pacientes embarazadas con anemia del HVCM. Cuenca,2018

\begin{tabular}{|c|c|c|c|c|c|c|c|}
\hline \multirow{3}{*}{ Factores asociados } & \multicolumn{7}{|c|}{ Anemia } \\
\hline & \multicolumn{2}{|c|}{$\mathbf{S i}$} & \multicolumn{2}{|c|}{ No } & \multirow[b]{2}{*}{$\mathbf{R P}$} & \multirow[b]{2}{*}{ IC $95 \%$} & \multirow[b]{2}{*}{ Valor $\mathrm{p}$} \\
\hline & $\mathbf{n}$ & $\%$ & $\mathbf{n}$ & $\%$ & & & \\
\hline \multicolumn{8}{|l|}{ Edad } \\
\hline Juventud & 51 & 34,2 & 98 & 65,8 & \multirow{2}{*}{1,7} & \multirow{2}{*}{$1,2-2,5$} & \multirow{2}{*}{0.00} \\
\hline Adulto & 40 & 19,5 & 165 & 80,5 & & & \\
\hline \multicolumn{8}{|l|}{ Residencia } \\
\hline Rural & 47 & 27,8 & 122 & 72,2 & \multirow{2}{*}{1,1} & \multirow{2}{*}{$0,8-1,4$} & \multirow{2}{*}{0,74} \\
\hline Urbana & 44 & 23,8 & 141 & 72,2 & & & \\
\hline \multicolumn{8}{|l|}{ Instrucción } \\
\hline Ninguna/Primaria & 26 & 22,6 & 89 & 77,4 & \multirow{2}{*}{0,8} & \multirow{2}{*}{$0,5-1,2$} & \multirow{2}{*}{0,35} \\
\hline Secundaria/Superior & 65 & 27,2 & 174 & 72,8 & & & \\
\hline \multicolumn{8}{|l|}{ Controles prenatales } \\
\hline Menos de 5 & 23 & 2,4 & 72 & 75,8 & \multirow{2}{*}{0,9} & \multirow{2}{*}{$0,6-1,3$} & \multirow{2}{*}{0,69} \\
\hline Más de 5 & 68 & 26,2 & 191 & 73,8 & & & \\
\hline \multicolumn{8}{|l|}{ Edad gestacional } \\
\hline A término & 79 & 25,5 & 231 & 74,5 & \multirow{2}{*}{0,9} & \multirow{2}{*}{$0,9-1$} & \multirow{2}{*}{0,79} \\
\hline Pretérmino & 12 & 27,3 & 32 & 72,8 & & & \\
\hline \multicolumn{8}{|l|}{ Gestación } \\
\hline Primigesta & 34 & 34,7 & 64 & 65,3 & \multirow{2}{*}{1,5} & & \\
\hline Multigesta & 57 & 22,3 & 199 & 77,7 & & $1-2,1$ & 0,01 \\
\hline Aborto & & & & & & & \\
\hline $\mathrm{Si}$ & 11 & 18 & 50 & 82 & 06 & $03-11$ & 013 \\
\hline No & 80 & 27,3 & 213 & 72,7 & 0,6 & $0,3-1,1$ & 0,13 \\
\hline Cesárea & & & & & & & \\
\hline $\mathrm{Si}$ & 22 & 25,3 & 65 & 74,7 & & & \\
\hline No & 69 & 25,9 & 198 & 74,1 & 0,9 & $0,6-1,4$ & 0,91 \\
\hline Parto vaginal & & & & & & & \\
\hline $\mathrm{Si}$ & 42 & 24,6 & 129 & 75,4 & & & \\
\hline No & 49 & 26,7 & 134 & 73,3 & 0,9 & $0,7-1,2$ & 0,36 \\
\hline Estado nutricional & & & & & & & \\
\hline Sobrepeso + Obesidad & 71 & 23,8 & 227 & 76,2 & & & \\
\hline Normal & 20 & 37 & 34 & 63 & 0,6 & $0,4-0,9$ & 0,04 \\
\hline
\end{tabular}

\section{Discusión}

Se estudiaron 354 gestantes que acudieron al Hospital Vicente Corral Moscoso cursando el tercer trimestre de embarazo, donde se encontró una prevalencia de anemia del $25,7 \%$ (IC $21.01 \%-30.4 \%$ ), datos similares se obtuvieron en un metaanálisis realizado en China con un total de 58752 que determinaron una prevalencia del $19,9 \%$ y en el tercer trimestre de $28,1 \%,{ }^{13}$ este estudio, que por su nivel de evidencia mostró resultados muy consistentes expuso una prevalencia muy cercana a la presentada en la población de Cuenca.

En comparación con otros estudios, presenta diferencias significativas, tal es el caso de Rosas y colaboradores ${ }^{29}$ quienes en México encontraron una prevalencia del $13 \%$, es decir casi un $50 \%$ menos que en nuestra población, esta diferencia puede deberse a varios factores uno de ellos es el nivel de obesidad elevado. Otro estudio realizado en Perú en el 2018, determinó una prevalencia de anemia en el tercer trimestre del embarazo de $43,6 \%$, en 172 pacientes, ${ }^{30}$ es evidente que la frecuencia de esta patología es muy variable y depende de varios factores, en este caso en Perú la prevalencia es casi el doble de la reportada en Cuenca.
Moyolema, ${ }^{31}$ estudió 92 casos de anemia, se clasificó los niveles de hemoglobina, leve $56 \%$, moderado $29 \%$; y severo $15 \%$, en nuestro estudio encontramos datos similares anemia leve $76,9 \%$, moderada $20.9 \%$ y severa en un $2,2 \%$. En un estudio en el Ecuador durante el 2017, utilizaron el volumen corpuscular medio para poder clasificar a la anemia según su morfología, se obtuvo como resultado un $59 \%$ VCM bajo, $25 \%$ alto y $16 \%$ normal. Contrarrestando los datos de nuestro estudio en el que encontramos la mayor frecuencia correspondió a normocítica con un 90,1\%, microcítica $8.8 \%$ y macrocítica $1,1 \%$.

Soto, ${ }^{32}$ asoció a la edad como factor de riesgo, observó que las gestantes menores de 30 años presentaron 2,2 veces más de riesgo que las mayores de 30 años (IC 95\% 1,17 $-4,48, p=0,01)$, con lo cual demostró que existía significancia estadística, en nuestro estudio se encontró que las pacientes en el grupo de edad juventud con una media de 26 años aumenta el riesgo de anemia en 1,7 veces, siendo este resultado estadísticamente significativo (IC 95\% 1,17 $-4,48, \mathrm{p}=0,01)$.

La residencia de mayor frecuencia fue en el área rural, y este factor no fue estadísticamente significativo al momento 
de analizarlo con anemia $(\mathrm{p}=0,74)$, demostrando que la distribución de los datos es al azar. Al respecto se menciona que estudios como el de Jiang y colaboradores ${ }^{33}$ y Lin y colaboradores $^{34}$ encontraron datos similares en el sentido de que las mujeres habitantes del área rural poseen mayor riesgo de anemia.

En cuanto a la prevalencia de anemia según el nivel de instrucción, no se demostró significancia estadística por el valor de $\mathrm{p}=0,35$; al respecto Yilmaz y colaboradores ${ }^{35}$ tampoco encontraron significancia estadística $(\mathrm{p}=0,96)$, pero se resalta que las mujeres con bajo nivel de instrucción poseen mayor frecuencia de anemia.

Abanto y colaboradores ${ }^{36}$ apoyan lo expuesto pues mencionan que las mujeres con nivel de instrucción analfabeta/primaria poseen 1,53 más riesgo de anemia, datos que fueron corroborados por Huanco y colaboradores. ${ }^{37}$

En lo que respecta a los controles prenatales, en este estudio la población que tenía más de 5 controles prenatales presentó un $74,7 \%$ de anemia, Soto ${ }^{32}$ en su estudio expone que las gestantes que no presentaron controles prenatales tienen mayor porcentaje de anemia $64,9 \%$, además el $\mathrm{OR}=0,03$ para la variable más de 5 controles y teniendo como valores de IC $95 \%(0,01-0,06)$, se asume que los controles prenatales es un factor protector para que las gestantes no tengan anemia, y con un valor de $\mathrm{p}=0,00$ demostrando con esto que hay significancia estadística.

Los datos encontrados en este estudio difieren de lo expuesto por Soto, ${ }^{32}$ este autor mostró una relación inversamente proporcional entre el número de controles y anemia mientras que en nuestro estudio es directamente proporcional, al respecto se debe anotar que no se dispone de una variable que indique el seguimiento a las pacientes durante su embarazo, una paciente con más de 5 controles pudo haber tenido los 5 controles en un periodo corto y no existió el seguimiento adecuado, a esto se suma que no existe garantía que los controles prenatales y las indicaciones enviadas en los mismos sean cumplidos por las embarazadas, lo que limita el análisis de este factor.

Bustos $^{23}$ encontró a la anemia como factor de riesgo asociado a la amenaza de parto pretérmino con OR 2,53, IC $95 \%(1,39-4,61)$, y con un valor de $\mathrm{P}<0,002$, por lo cual se comprueba la asociación entre las dos variables en este estudio. Sin embargo, en nuestro estudio no se logró establecer dicha asociación pues el valor de X2 y p no fueron estadísticamente significativos.

Otro estudio en el área del Mediterráneo en el Sur de Europa, determinó en el año 2018 que la anemia en el primer trimestre del embarazo fue de $3,8 \%$ pero aumentó hasta un $21,5 \%$ en el tercer trimestre, ${ }^{38}$ en donde las condiciones socioeconómicas son mejores que en Ecuador, sin embargo, la prevalencia de anemia fue casi similar, lo cual nos lleva a considerar la presencia de otros factores asociados.

Por otra parte en Etiopía en el año 2017, se determinó la prevalencia de anemia en $23 \%$ y conforme se analizó los factores que se relacionan estos son; el nivel socio económi- co, el cursar el tercer trimestre del embarazo, multiparidad, bajo acceso a cuidados prenatales y malnutrición. ${ }^{14} \mathrm{Si}$ bien es cierto, este estudio probablemente por las diferencias poblacionales no sea comparable con la población de Cuenca, es evidente que los factores si lo son: en especial el nivel socio económico que denota un mayor riesgo de anemia ante la pobreza y el cursar el tercer trimestre del embarazo.

En el estudio de Soto, ${ }^{32}$ en cuanto a la paridad de las mujeres, las gestantes multíparas presentaron anemia en mayor porcentaje con el $61,7 \%$ con un valor de $\mathrm{p}=0,03$ y $\mathrm{OR}=1,83$ IC $95 \%(1,04-3,21)$, ya que el periodo intergenésico entre algunas de ellas era corto, menor a 2 años, por lo que les convierte en un factor de riesgo para desarrollar anemia, por otro lado en nuestro estudio se determinó que las pacientes primigestas presentaban RP 1,5 (IC:95\% 1-2,1), más de riesgo de desarrollar anemia durante el embarazo que las multigestas. Este estudio se puede comparar con los resultados de Ortiz y colaboradores, ${ }^{39}$ quienes encontraron que las pacientes con mayor cantidad de partos poseen un factor protector de 0,87 para la presentación de anemia en comparación con primigestas que poseen mayor riesgo de anemia.

En lo que respecta a los antecedentes gineco-obstétricos, en este estudio no se determinó asociación entre aborto, cesárea y parto vaginal con anemia. La frecuencia de anemia en mujeres con antecedente de cesárea fue de $24,2 \%$, parto vaginal $46,2 \%$ y aborto el $12,1 \%$. Al respecto Flores y colaboradores $^{40}$ en el 2019 exponen que las mujeres con ninguna cesárea presentan hasta un $69 \%$ y las que presentan al menos 1 cesárea el $31 \%$; estos datos presentan una relación similar a la de nuestro estudio, pero el impacto de estas vías de terminación del parto es mayor para las pacientes con parto normal.

Concerniente al estado nutricional, en este estudio se presentó un 40,7\% de sobrepeso y $37,4 \%$ de obesidad, al analizar estas variables con anemia se encontró que la obesidad/sobrepeso es un factor protector para anemia RP 0,6 (IC 95\% 0,4-0,9), que coincide con un estudio de China $^{41}$ en el 2018, con un total de 11782 mujeres, donde se determinó que el sobrepeso y la obesidad son un factor protector para anemia, para sobrepeso OR 0,68 y 0,30 para obesidad. Los datos de prevalencia de sobrepeso y obesidad se contraponen con los expuestos por Agrawal y $\mathrm{Singh}^{42}$ quien demostró en un estudio con 1000 gestantes que la frecuencia de obesidad es de $17 \%$ y sobrepeso de $18 \%$; estos datos difieren de los valores encontrados en Cuenca.

\section{Conclusiones}

En relación a las características sociodemográficas se presentó con mayor prevalencia en adultas jóvenes, sin encontrar significancia estadística con la residencia, ocupación, instrucción y estado civil.

Se presentó en embarazadas con $>5$ controles prenatales, en gestaciones a término y con sobrepeso. 
De las 91 pacientes con anemia los factores asociados que se presentaron con mayor frecuencia fueron: la edad de juventud plena, en zonas rurales, de instrucción secundaria, ocupación QQDD, estado civil soltera y casadas con $>5$ controles prenatales, con sobrepeso y en relación a los antecedentes obstétricos no se encontró significancia estadística.

Según la clasificación de la anemia por la gravedad se presentó con mayor frecuencia la anemia leve con una morfología normocítica.

Se comportaron como factores de riesgo el grupo de edad considerado como juventud y las pacientes primiges- 10 . tas, el estado nutricional sobrepeso/obesidad se comportó como factor protector para anemia.

\section{Fuente de Financiamiento}

Este estudio es autofinanciado.

\section{Conflicto de Intereses}

No existen conflictos personales, profesionales, financieroso de otro tipo.

\section{Consentimiento Informado}

Los autores cuentan con el consentimiento informado de los pacientes para la investigación, la publicación del caso y sus imágenes.

\section{Referencias Bibliográficas}

1. Pavord S, Daru J, Prasannan N, Robinson S, Stanworth $\mathrm{S}$, Girling J, et al. UK guidelines on the management of iron deficiency in pregnancy. Br J Haematol [Internet]. 2 de octubre de 2019 [citado 8 de febrero de 2020]; Disponible en: https://onlinelibrary.wiley.com/doi/abs/10. 1111/bjh.16221

2. Milman N. Fisiopatología e impacto de la deficiencia de hierro y la anemia en las mujeres gestantes y en los recién nacidos/infantes. Rev Peru Ginecol Obstet. 2012;58(4):293-312.

3. Kumar KJ, Asha N, Murthy DS, Sujatha M, Manjunath V. Maternal anemia in various trimesters and its effect on newborn weight and maturity: an observational study. Int J Prev Med. febrero de 2013;4(2):193-9.

4. Aueberbach M, Landy H. Anemia in pregnancy [Internet]. UpToDate; 2020 [citado 8 de febrero de 2020]. Disponible en: https://www.uptodate.com/contents/anemia-inpregnancy?search=Anemia\%20in\%20pregnancy $\%$ 2FMichael\%20Auerbach\&source=search_result\& selectedTitle $=1 \sim 150 \&$ usage_type $=$ default $\&$ display_ rank $=1$

5. Organización Mundial de la Salud. Sistema de Información Nutricional sobre Vitaminas y Minerales (VMNIS). Prevalencia mundial de la anemia y número de personas afectadas [Internet]. 2008 [citado 8 de febrero de 2020]. Disponible en: https://www.who.int/vmnis/ database/anaemia/anaemia_data_status_t2/es/
6. ACOG Practice Bulletin No. 95: Anemia in Pregnancy: Obstet Gynecol. julio de 2008;112(1):201-7.

7. Ministerio de Salud Pública. Diagnóstico y tratamiento de la anemia en el embarazo: Guía de práctica clínica. 2014.

8. San Gil C, Villazán C, Ortega Y. Characterization of anemia during pregnancy and some associated risk factors in pregnant women at Regla municipality. Rev Cuba Med Gen Integral. 30(1):71-81.

9. De La Oz F, Orozco L. Anemia en el embarazo, un problema de salud que puede prevenirse. Medicas UIS. 26(3):45-50.

. Erez Azulay C, Pariente G, Shoham-Vardi I, Kessous R, Sergienko R, Sheiner E. Maternal anemia during pregnancy and subsequent risk for cardiovascular disease. J Matern Fetal Neonatal Med. 13 de octubre de 2015;28(15):1762-5.

11. García A, Izaguirre D, Álvarez D. Impacto de la anemia para una embarazada e importancia del riesgo preconcepcional. Rev Cuba Med Gen Integral. 2017;33(1):146-53.

12. Mohamed MA, Ahmad T, Macri C, Aly H. Racial disparities in maternal hemoglobin concentrations and pregnancy outcomes. J Perinat Med [Internet]. 1 de enero de 2012 [citado 8 de febrero de 2020];40(2). Disponible en: https://www.degruyter.com/view/j/jpme.2012.40. issue-2/jpm.2011.137/jpm.2011.137.xml

13. Zhao S, Jing W, Liu J, Liu M. Prevalence of anemia during pregnancy in China, 2012-2016: a Meta-analysis [Internet]. Zhonghua Yu Fang Yi Xue Za Zhi; 2018 [citado 18 de enero de 2020]. Disponible en: https://www.ncbi. nlm.nih.gov/pubmed/30196645

14. Lebso M, Anato A, Loha E. Prevalence of anemia and associated factors among pregnant women in Southern Ethiopia: A community based cross-sectional study. Clarke SL, editor. PLOS ONE. 11 de diciembre de 2017;12(12):e0188783.

15. Rincón-Pabón D, Urazán-Hernández Y, GonzalezSantamaria J. Prevalencia y factores sociodemográficos asociados a anemia ferropénica en mujeres gestantes de Colombia (análisis secundario de la ENSIN 2010). Nutr Hosp [Internet]. 2018 [citado 8 de febrero de 2020]; Disponible en: https://www.nutricionhospitalaria.org/ articles/01895/show

16. Munares O, Gómez G, Barboza J, Sánchez J. Niveles de hemoglobina en gestantes atendidas en establecimientos del Ministerio de Salud del Perú, 2011. Rev Peru Med Exp Salud Publica. 2012;29(3):329-36.

17. Montoya J, Castelazo E, Valerio E, Velásquez G, Nava D, Escárcega J, et al. Review by expert group in the diagnosis and treatment of anemia in pregnant women. Federación Mexicana de Colegios de Obstetricia y Ginecología. Ginecol Obstet Mex. 2012;80(9):563-80.

18. Pérez M, Peralta M, Villalba Y, Vanegas S, Rivera J, Galindo J, et al. Caracterización de la población con anemia en el embarazo y su asociación con la morbimortalidad perinatal. Rev Médica Risaralda. 2019;25(1):33-9.

19. Cabezas D, Balderrama L, Borda V, Colque C, Jimé- 
nez M. Prevalencia de Anemia Nutricional en el Em-30. Cahuapaza F. Correlación entre anemia materna en el terbarazo, en centros de salud Sarcobamba y Solomon Klein Enero 2010 - 2011. Rev Científica Cienc Médica. 2012;15(1):11-3.

20. Fernández J, Rojas M, Bojorquez I, Giraldo V, Sobczyk R, Acosta J, et al. Situación de salud de gestantes migrantes venezolanas en el Caribe colombiano: primer reporte para 31. una respuesta rápida en Salud Pública. Rev Univ Ind Santander Salud. 2019;51(3):208-19.

21. Figueroa A, Chicaiza M. Prevalencia de Anemia Gestacional en pacientes con labor de parto y efecto en la reserva de hierro del recién nacido en el HGOIA [Internet]. [Tesis]. [Quito]: Universidad Central del Ecuador; 2012 [citado 5 de marzo de 2020]. Disponible en: http://www.dspace.uce. edu.ec/bitstream/25000/619/1/T-UCE-0006-19.pdf

22. Narváez J, Herrera RH, Orellana M, Gloria F. Prevalencia de Anemia con y sin Hemoglobina ajustada, en parturientas del Hospital Vicente Corral Moscoso.Rev Médica HJCA. 2012 Sep;4(2):181-5.

23. Bustos D, Galarza B. Anemia en la gestación y su relación con amenaza de parto pretérmino y parto pretérmino, en el Hospital San Vicente de Paul de la Ciudad de Ibarra 33. y Hospital Gustavo Dominguez de Santo Domingo de la Tsáchilas en el periodo enero a julio de 2017 [Internet] [Tesis]. [Quito]: Pontificia Universidad Católica del Ecuador; 2018 [citado 18 de enero de 2020]. Disponible 34 en: http://repositorio.puce.edu.ec/bitstream/handle/ 22000/14976/TESIS\%20CORREGIDA\%20DRA\% 20PALACIOS\%20DR\%20GALARZA\%20DRA\% 20BUSTOS.pdf? sequence $=1 \&$ isAllowed $=y$

24. Arispe C, Salgado M, Tang G, González C, Rojas J. Frecuencia de control prenatal inadecuado y de factores asociados a su ocurrencia. Rev Medica Hered. 2011;22(4):159-60.

25. Vaca A. Políticas y programas implementados en Ecuador para tratar las deficiencias de micronutrientes en los últimos 15 años [Internet] [Tesis]. Universidad San Francisco de Quito; 2015 [citado 8 de febrero de 2020]. Disponible en: http://repositorio.usfq.edu.ec/handle/23000/5292

26. Martínez A, Jaramillo L, Villegas J, Álvarez L, Ruiz C. La anemia fisiológica frente a la patológica en el embarazo. Rev Cuba Obstet Ginecol [Internet]. 2018 [citado 8 de febrero de 2020];44(2). Disponible en: http://www. revginecobstetricia.sld.cu/index.php/gin/article/view/356

27. Olukayode A. Hematological Changes in Pregnancy - 37 The Preparation for Intrapartum Blood Loss. Obstet Gynecol Int J [Internet]. 2016 [citado 8 de febrero de 2020];4(3). Disponible en: http://medcraveonline.com/ OGIJ/OGIJ-04-00109.php

28. Ministerio de Salud Pública. Diagnóstico y tratamiento de la anemia en el embarazo: Guía de práctica clínica. Quito: 38. Ribot B, Ruiz F, Abajo S, March G, Fargas F, Arija Ministerio de salud pública; 2014.

29. Rosas M, Ortíz M, Dávila R, González A. Prevalencia y factores predisponentes de anemia en el embarazo en una clínica de primer nivel. Rev Hematol Mex. 2016;17:10713. cer trimestre con el peso y hemoglobina del recién nacido en el Hospital EsSalud III Juliaca - enero a diciembre 2017 [Internet] [Tesis]. [Perú]: Universidad Nacional del Altiplano; 2018 [citado 18 de enero de 2020]. Disponible en: http://repositorio.unap.edu.pe/handle/UNAP/6374

. Moyolema Y. Incidencia de anemia en gestantes atendidas en la consulta externa de un Hospital GinecoObstétrico de la ciudad de Guayaquil desde octubre del 2016 a febrero del 2017 [Internet] [Trabajo de titulación]. [Guayaquil]: Universidad Católica de Santiago de Guayaquil; 2017 [citado 18 de enero de 2020]. Disponible en: http://repositorio.ucsg.edu.ec/bitstream/3317/ 7498/1/T-UCSG-PRE-MED-ENF-349.pdf

32. Soto J. Factores asociados a anemia en gestantes hospitalizadas en el servicio de ginecoobstetricia del Hospital "San José” Callao - Lima. 2018 [Internet] [Tesis]. [Lima]: Universidad Ricardo Palma; 2018 [citado 18 de enero de 2020]. Disponible en: http://repositorio. urp.edu.pe/bitstream/handle/URP/1256/161\%20SOTO\% 20RAMIREZ.pdf? sequence=1\&isAllowed=y

3. Jiang S, Pang XH, Duan YF, Bi Y, Wang J, Yin SA, et al. [The influencing factors of anemia for pregnant women between 2010-2012 in China]. Zhonghua Yu Fang Yi Xue Za Zhi. 6 de enero de 2018;52(1):21-5.

4. Lin L, Zhu W, Wang C, Su R, Feng H, Yang H. Prevalence, risk factors and associated adverse pregnancy outcomes of anaemia in Chinese pregnant women: a multicentre retrospective study. BMC Pregnancy Childbirth [Internet]. diciembre de 2018 [citado 8 de febrero de 2020];18(1). Disponible en: https://bmcpregnancychildbirth.biomedcentral.com/ articles/10.1186/s12884-018-1739-8

35. Yılmaz E, Yılmaz Z, Çakmak B, Gültekin İB, Çekmez Y, Mahmutoğlu S, et al. Relationship between anemia and depressive mood in the last trimester of pregnancy. J Matern Fetal Neonatal Med. 18 de abril de 2017;30(8):97782.

36. Abanto M, Salcedo M, Vásquez T. Anemia y factores de riesgo asociados en mujeres gestantes. Centro de Salud Patrona de Chota, 2017. 2017 [citado 8 de febrero de 2020];Caxamarca. Revista Científica de la Universidad Nacional de Cajamarca(16). Disponible en: http://190. 116.36.87/index.php/Caxamarca/article/view/21

37. Huanco D, Ticona M, Aguilar J, Gomez J. Incidencia y factores de riesgo de la anemia en el embarazo en el Hospital Hipolito Unanue de Tacna 2001-2010 [Internet]. Revista Médica Basadrina; 2019 [citado 8 de febrero de 2020]. Disponible en: http://revistas.unjbg.edu.pe/index. $\mathrm{php} / \mathrm{rmb} / \mathrm{article} / \mathrm{view} / 463$

V. Prevalence of anaemia, risk of haemoconcentration and risk factors during the three trimesters of pregnancy. Nutr Hosp [Internet]. 10 de enero de 2018 [citado 18 de enero de 2020]; Disponible en: http://revista. nutricionhospitalaria.net/index.php/nh/article/view/1045. 
39. Ortiz Montalvo YJ, Ortiz Romaní KJ, Castro Trujillo BS, Nuñez Revilla SC, Rengifo Balta GL. Factores sociodemográficos y prenatales asociados a la anemia en gestantes peruanas. Enferm Glob. 18 de septiembre de 2019; 18(4):273-90.

40. Flores S, Germes F, Levario M. Complicaciones obstétricas y perinatales en pacientes con anemia. Ginecol Obstet Mex. 2019;87(2):85-92.

41. Tan J, Qi Y-N, He G-L, Yang H-M, Zhang G-T, Zou $\mathrm{K}$, et al. Association between Maternal Weight Indicators and Iron Deficiency Anemia during Pregnancy: A Cohort Study. Chin Med J (Engl). noviembre de 2018;131(21):2566-74.

42. Agrawal S, Singh A. Obesity or Underweight-What is Worse in Pregnancy? J Obstet Gynecol India. diciembre de 2016;66(6):448-52.

Recibido: 7 de octubre de 2020

Aceptado: 21 de octubre de 2020

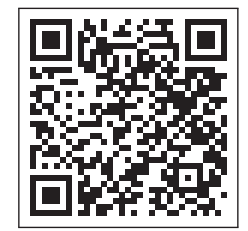


